\title{
On Electromagnetic-Acoustic Analogies in Energetic Relations for Waves Interacting with Material Surfaces
}

\author{
A. Dukata* AND J. KaPELEWSKI \\ Faculty of Electronics, Military University of Technology \\ S. Kaliskiego 2, 00-908 Warsaw, Poland
}

In the paper we consider propagation of a monochromatic surface electromagnetic field at the interface separating two semi-infinite media - a metamaterial (generally with complex permittivity and permeability) and a conventional dielectric medium. The metamaterial is treated as homogeneous and isotropic (it is so-called effective medium approximation). It is true for e.g. artificial metamaterials with negative dielectric permittivity and negative permeability in the microwave frequency range. The last materials are known as left handed and a conventional as right handed media. In this work the classical optical theorem is extended to the case of scattering of electromagnetic surface waves incident at the interface separating a left handed medium and a right handed dielectric medium. The analogous theorem is known in the scattering theory for elastic fields.

PACS numbers: 41.20.Jb

\section{Introduction}

The dynamic field problems, such as uniform plane wave propagation, reflection, scattering and guided waves, of greatest interest in acoustics are of the similar nature as problems in electromagnetism. The basic philosophy of the paper stems from the inherent analogy of surface and interface waves of both acoustics (see $[1,2]$ and electromagnetic (EM) type.

The interface plane EM modes may be considered as a portion of the complete spectrum representation of waves (usually called complex waves, see e.g. [3]). Generally, there are two independent interface modes - TE-polarized (transverse electric) and TM-polarized (transverse magnetic).

The interface TM modes might be utilized e.g. in the high-frequency surface-wave radars (HFSWR). The integrated maritime surveillance system based on

${ }^{*}$ corresponding author; e-mail: andrzej.dukata@wel.wat.edu.pl 
HFSWR provides over-the-horizon surveillance of large ocean areas, out to 200 nautical mile [4].

Our main objective in this article is to formulate an extended optical theorem (or cross-section theorem) for surface EM waves scattering by an obstacle embedded in the near interface region.

Our results follow from the consideration of the form of incident and scattered EM fields in Sect. 3. In Sect. 4 we derive the main result by using energy conservation concepts and the method of stationary phase.

\section{Description of the configuration}

Without loss of generality, we consider an interface with an embedded obstacle as shown in Fig. 1. The obstacle occupies the domain inside a sufficiently regular, bounded, closed surface $S_{\mathrm{ob}}$.

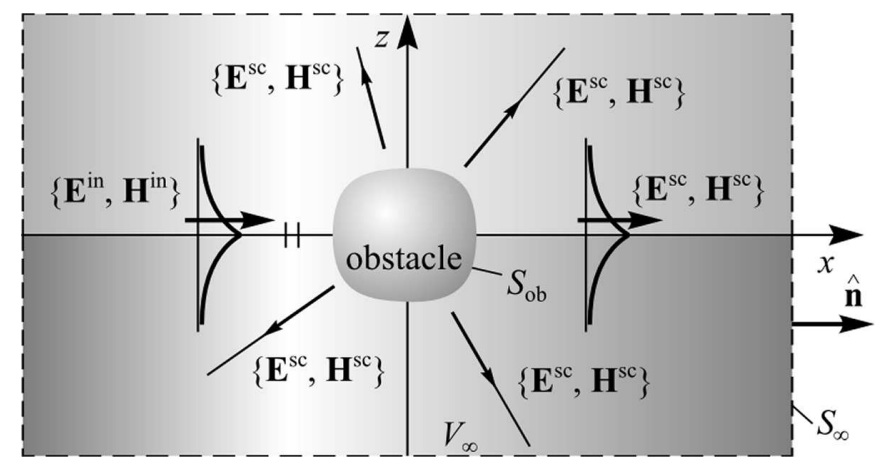

Fig. 1. An obstacle embedded in near-surface region of an interface between two different media is illuminated by an incident EM surface wave.

The domain exterior to $S_{\mathrm{ob}}$ is called $V_{\infty}$. It is described by the electric permittivity $\varepsilon_{\alpha}$ and magnetic permeability $\mu_{\alpha}$, where $\alpha=$ I, II for medium I and II, respectively. The region above the interface (denoted by I) is an ordinary material (e.g. homogeneous, lossless, and isotropic) and both $\varepsilon_{\mathrm{I}}$ and $\mu_{\mathrm{I}}$ are real and positive. The region below the interface is a homogeneous and isotropic metamaterial with complex $\varepsilon_{\text {II }}$ and $\mu_{\text {II }}$. Let $S_{\infty}$ be the cylindrical surface of infinite radius with the outward unit vector $\hat{\boldsymbol{n}}$ normal to the surface. In $V_{\infty}$ an EM state is characterised by its electric $\boldsymbol{E}$ and magnetic field $\boldsymbol{H}$.

We consider the case where the incident wave $\left\{\boldsymbol{E}^{\text {in }}, \boldsymbol{H}^{\text {in }}\right\}$ is a plane surface wave. The presence of perturbation in material properties produces the scattered field $\left\{\boldsymbol{E}^{\mathrm{sc}}, \boldsymbol{H}^{\mathrm{sc}}\right\}$. It can be considered as the difference between the actual field and the incident field $\left\{\boldsymbol{E}^{\text {sc }}, \boldsymbol{H}^{\text {sc }}\right\} \stackrel{\text { def }}{=}\left\{\boldsymbol{E}-\boldsymbol{E}^{\text {in }}, \boldsymbol{H}-\boldsymbol{H}^{\text {in }}\right\}$. 


\section{Properties of incident and scattered surface waves}

\subsection{Incident field}

Let us consider a plane, monochromatic wave propagating in the $x$ direction along the interface between semi-infinite media I and II as shown in Fig. 1. At all the equations the factor $\exp (-\mathrm{i} \omega t)$ is omitted but understood. Since this is a $2 \mathrm{D}$ problem with $\partial / \partial y=0$, it is well known that there are two independent modes, TE and TM. We introduce the cylindrical coordinate system $\hat{\boldsymbol{r}}, \hat{\boldsymbol{\theta}}, \hat{\boldsymbol{z}}$. If the appropriate $z$-component of an incident plane wave has the standard form

$$
U^{\text {in }} \exp \left(\mathrm{i} k x-q_{\alpha}|z|\right)= \begin{cases}B_{z} & \text { for } \mathrm{TE} \\ D_{z} & \text { for } \mathrm{TM}\end{cases}
$$

the components of TE mode are given in terms of $B_{z}$ :

$$
\begin{aligned}
& E_{r}=\frac{\omega}{k} B_{z} \sin \theta, \quad E_{\theta}=\frac{\omega}{k} B_{z} \cos \theta, \quad E_{z}=0 \\
& H_{r}=-\mathrm{i} \frac{1}{\mu_{\alpha}} \frac{\operatorname{sgn}(z) q_{\alpha}}{k} B_{z} \cos \theta, \quad H_{\theta}=\mathrm{i} \frac{1}{\mu_{\alpha}} \frac{\operatorname{sgn}(z) q_{\alpha}}{k} B_{z} \sin \theta, \quad H_{z}=\frac{1}{\mu_{\alpha}} B_{z}
\end{aligned}
$$

and the components of TM mode are given by $D_{z}$ :

$$
\begin{aligned}
& E_{r}=-\mathrm{i} \frac{1}{\mu_{\alpha}} \frac{q_{\alpha} \operatorname{sgn}(z)}{k} D_{z} \cos \theta, \quad E_{\theta}=\mathrm{i} \frac{1}{\mu_{\alpha}} \frac{q_{\alpha} \operatorname{sgn}(z)}{k} D_{z} \sin \theta, \quad E_{z}=\frac{1}{\varepsilon_{\alpha}} D_{z}, \\
& H_{r}=-\frac{\omega}{k} D_{z} \sin \theta, \quad H_{\theta}=-\frac{\omega}{k} D_{z} \cos \theta, \quad H_{z}=0 .
\end{aligned}
$$

In the above equations the $U^{\text {in }}$ is the wave amplitude, $k$ is a propagation constant, $q_{\alpha}=\left(k^{2}-k_{\alpha}^{2}\right)^{1 / 2}$ is real and positive transverse wave number which characterises the inverse decay length of the surface wave in the corresponding medium and $k_{\alpha}^{2}=\omega^{2} \varepsilon_{\alpha} \mu_{\alpha}$. Moreover, the boundary conditions give the dispersion relations $q_{\mathrm{I}} / \mu_{\mathrm{I}}+q_{\mathrm{II}} / \mu_{\mathrm{II}}=0$ and $q_{\mathrm{I}} / \varepsilon_{\mathrm{I}}+q_{\mathrm{II}} / \varepsilon_{\mathrm{II}}=0$ for the TE- and TM-polarized surface waves, respectively.

\subsection{Scattered field}

Let us consider now all monochromatic solutions of Maxwell's equations in the form

$$
\begin{aligned}
& \boldsymbol{E}_{\alpha}(x, y, z)=\boldsymbol{E}_{\alpha}(x, y) \exp \left(-q_{\alpha}|z|\right), \\
& \boldsymbol{H}_{\alpha}(x, y, z)=\boldsymbol{H}_{\alpha}(x, y) \exp \left(-q_{\alpha}|z|\right) .
\end{aligned}
$$

This is a $2 \mathrm{D}$ problem with two independent modes TE and TM, too. In the polar coordinate system $\hat{\boldsymbol{r}}, \hat{\boldsymbol{\theta}}$ the motion of media is governed by the scalar equation

$$
\left(\frac{\partial^{2} \psi}{\partial r^{2}}+\frac{1}{r} \frac{\partial \psi}{\partial r}+\frac{1}{r^{2}} \frac{\partial^{2} \psi}{\partial \theta^{2}}+k_{\alpha}^{2}+q_{\alpha}^{2}\right) \psi(r, \theta) ; \quad \psi=\left\{\begin{array}{cc}
B_{z} & \text { for TE mode } \\
D_{z} & \text { for TM mode }
\end{array}\right.
$$

The required scattered field is the asymptotic form of $\psi(r, \theta)$ for $|k r|=1$ 


$$
\psi(r, \theta)=\frac{\exp \left(\mathrm{i} k r+\frac{1}{4} \mathrm{i} \pi\right)}{\sqrt{\frac{1}{2} \pi k r}} A^{\mathrm{sc}}(\theta)
$$

where $A^{\mathrm{sc}}(\theta)$ is the scattering amplitude. Then $E_{r}=0, E_{\theta}=k_{\frac{\omega}{k_{\alpha}^{2}+q_{\alpha}^{2}}} B_{z}$, $H_{r}=-\frac{\mathrm{i} k}{\mu_{\alpha}} \frac{q_{\alpha} \operatorname{sgn}(z)}{k_{\alpha}^{2}+q_{\alpha}^{2}} B_{z}, \quad H_{\theta}=0$ for TE mode and $E_{r}=-\frac{\mathrm{i} k}{\varepsilon_{\alpha}} \frac{q_{\alpha} \operatorname{sgn}(z)}{k_{\alpha}^{2}+q_{\alpha}^{2}} D_{z}$, $E_{\theta}=0, H_{r}=0, H_{\theta}=-k \frac{\omega}{k_{\alpha}^{2}+q_{\alpha}^{2}} D_{z}$ for TM mode.

\section{Power-flow relations and optical theorem}

The time-averaged Poynting vector associated with the electromagnetic wave motion is given by

$$
\langle\boldsymbol{S}\rangle_{T}=\frac{1}{2} \operatorname{Re}\left\{\boldsymbol{E} \times \boldsymbol{H}^{*}\right\},
$$

where $\operatorname{Re}($ ) denotes the real part of its argument, and the superscript " "* denotes the complex conjugate operator. Because the evanescent waves do not contribute to the far field, we restrict ourselves to the case when the both half spaces are non-absorbing, but the second has negative refractive index. It is worth to note that the TE- and TM-polarized waves cannot coexist simultaneously for the same set of parameters [5].

Applying energy conservation concept, the sum of a time-averaged power flow carried by the total field across the closed boundary $S_{\infty}$ of the domain $V_{\infty}$ and an average power $P^{a}$ being absorbed by the scatterer is equal to zero

$$
\int_{S_{\infty}}\langle\boldsymbol{S}\rangle_{T} \cdot \hat{\boldsymbol{n}} \mathrm{d} A+P^{\mathrm{a}}=0
$$

Hence, for incident TE or TM surface wave,

$$
P^{\mathrm{in}}+P^{\mathrm{is}}=-P^{\mathrm{a}}-P^{\mathrm{sc}}
$$

where

$$
\begin{aligned}
& P^{\mathrm{in}}=\frac{1}{2} \operatorname{Re}\left\{\int_{S_{\infty}}\left(\boldsymbol{E}^{\mathrm{in}} \times \boldsymbol{H}^{\mathrm{in} \mathrm{in}^{*}}\right) \cdot \hat{\boldsymbol{n}} \mathrm{d} A\right\} \equiv 0, \\
& P^{\mathrm{sc}}=\frac{1}{2} \operatorname{Re}\left\{\int_{S_{\infty}}\left(\boldsymbol{E}^{\mathrm{sc}} \times \boldsymbol{H}^{\mathrm{sc}^{*}}\right) \cdot \hat{\boldsymbol{n}} \mathrm{d} A\right\}, \\
& P^{\mathrm{is}}=\frac{1}{2} \operatorname{Re}\left\{\int_{S_{\infty}}\left(\boldsymbol{E}^{\mathrm{sc}} \times \boldsymbol{H}^{\mathrm{in} \mathrm{in}^{*}}+\boldsymbol{E}^{\mathrm{in}} \times \boldsymbol{H}^{\mathrm{sc}}\right) \cdot \hat{\boldsymbol{n}} \mathrm{d} A\right\} .
\end{aligned}
$$

At above equations $P^{\mathrm{sc}}$ represents the average total power scattered by the obstacle. The term $P^{\text {is }}$ contains contributions from the interaction between the incident plane wave and the scattered waves.

The last integral can be evaluated asymptotically by using the method of stationary phase. It can be shown that

$$
P^{\text {is }}=-\frac{\omega}{k^{2}} \operatorname{Im}\left[U^{\mathrm{in}^{*}} A^{\mathrm{sc}}(\theta=0)\right] \begin{cases}1 / \mu_{\mathrm{I}} q_{\mathrm{I}}+1 / \mu_{\mathrm{II}} q_{\mathrm{II}} & \text { for TE, } \\ 1 / \varepsilon_{\mathrm{I}} q_{\mathrm{I}}+1 / \varepsilon_{\mathrm{II}} q_{\mathrm{II}} & \text { for TM. }\end{cases}
$$


In these calculations we have assumed that there are no additional scattered "volume wave" contributions to radiated power in the far field.

We now introduce the scattering $\sigma^{\text {sc }}$ and absorption $\sigma^{\text {a }}$ cross-section of the obstacle for an incident surface wave by means of the formulae

$$
\sigma^{\mathrm{a}}=P^{\mathrm{a}} / P_{0}, \quad \sigma^{\mathrm{sc}}=P^{\mathrm{sc}} / P_{0},
$$

where $P_{0}$ is a linear power density of uniform surface wave

$$
P_{0}=\frac{\omega}{4 k}\left|U^{\mathrm{in}}\right|^{2} \begin{cases}1 / \mu_{\mathrm{I}} q_{\mathrm{I}}+1 / \mu_{\mathrm{II}} q_{\mathrm{II}} & \text { for TE, } \\ 1 / \varepsilon_{\mathrm{I}} q_{\mathrm{I}}+1 / \varepsilon_{\mathrm{II}} q_{\mathrm{II}} & \text { for TM. }\end{cases}
$$

Using the definitions and substituting it into equation of energy balance we find the optical theorem for an incident surface TE or TM wave

$$
\sigma^{\mathrm{t}}=\sigma^{\mathrm{sc}}+\sigma^{\mathrm{a}}=\frac{4}{k\left|U^{\mathrm{in}}\right|^{2}} \operatorname{Im}\left[U^{\mathrm{in}{ }^{*}} A^{\mathrm{sc}}(\theta=0)\right] .
$$

\section{Conclusions}

The optical theorem for an obstacle in an interface separating lossless $\mathrm{RH}-\mathrm{LH}$ media has been derived for incident TE- and TM-polarized surface waves. Results show that the knowledge of far-field scattering amplitude in the direction of the incident wave is sufficient to determine extinction properties of such a scatterer. If a perpendicular to the interface component of the incident and scattered fields are presented in the forms given by Eq. (1) and Eq. (2), respectively, then the resulting optical theorem has the standard form (3). We have derived the analogous simple form in our previous work for an incident surface elastic (Rayleigh) wave [1].

We have discussed the theorem applicability elsewhere (see e.g. [1]).

\section{Acknowledgments}

This paper is financed from sources for science in the years 2007-2010 in the frame of ordered scientific project PBZ-MNiSW-DBO-04/I/2007.

\section{References}

[1] A. Dukata, Ph.D. Thesis, Military University of Technology, Warsaw 2007 (in Polish).

[2] A. Dukata, J. Kapelewski, Biuletyn WAT LVI, 2, 123 (2007) (in Polish).

[3] A. Ishimaru, Electromagnetic Wave Propagation Radiation and Scattering, Prentice Hall, London 1991.

[4] L. Sevgi, A. Pansford, H.C. Chan, IEEE Ant. Prop. Mag. 43, 28 (2001).

[5] I. Sharidov, A. Sukhorukov, Y.S. Kivshar, Phys. Rev. E 69, 016617 (2004). 